\title{
Transmission blocking vaccines to control insect-borne diseases - A Review
}

\author{
Iliano V Coutinho-Abreu, Marcelo Ramalho-Ortigao/ ${ }^{+}$ \\ Biology of Disease Vectors Laboratory, Department of Entomology, Kansas State University, Manhattan, KS 66506, USA
}

\begin{abstract}
Insect-borne diseases are responsible for severe mortality and morbidity worldwide. As control of insect vector populations relies primarily on the use of insecticides, the emergence of insecticide resistance as well to unintended consequences of insecticide use pose significant challenges to their continued application. Novel approaches to reduce pathogen transmission by disease vectors are been attempted, including transmission-blocking vaccines (TBVs) thought to be a feasible strategy to reduce pathogen burden in endemic areas. TBVs aim at preventing the transmission of pathogens from infected to uninfected vertebrate host by targeting molecule(s) expressed on the surface of pathogens during their developmental phase within the insect vector or by targeting molecules expressed by the vectors. For pathogen-based molecules, the majority of the TBV candidates selected as well as most of the data available regarding the effectiveness of this approach come from studies using malaria parasites. However, TBV candidates also have been identified from midgut tissues of mosquitoes and sand flies. In spite of the successes achieved in the potential application of TBVs against insect-borne diseases, many significant barriers remain. In this review, many of the TBV strategies against insect-borne pathogens and their respective ramification with regards to the immune response of the vertebrate host are discussed.
\end{abstract}

Key words: transmission-blocking vaccines - insect and vector-borne disease

Insect-borne diseases cause about 1.5 million human deaths every year (Hill et al. 2005). Besides mortality, morbidity resulted from infection with such diseases responds for major economic losses mainly in developing countries. The burden of insect-borne diseases such as malaria and leishmaniasis in disability adjusted life years (DALY) reach almost 50 million, an indication that current strategies are not effective and new tools are necessary to combat the spread of such diseases (Hill et al. 2005). Among the many different strategies targeting disease vectors or the pathogens they transmit (Valenzuela 2004, Thomas \& Read 2007, Billingsley et al. 2008, Coutinho-Abreu et al. 2009, McMeniman et al. 2009, Oliveira et al. 2009), transmission-blocking vaccines (TBVs) are thought to represent a significant step in that direction and have now moved from the bench top to clinical trials (Saul et al. 2007, Wu et al. 2008).

TBVs aim at interfering and/or blocking pathogen development within the vector, halting transmission to non-infected vertebrate host (Figure). TBVs usually rely on immunization of vertebrate hosts (either infected or uninfected) with molecules derived from the pathogen

Financial support: National Institutes of Allergy and Infectious Diseases, National Institutes of Health (7RO1AI074691-02, 5R01AI074691-03), Kansas Agricultural Experiment Station (to MRO), Department of Entomology (to IVC-A), Kansas State University This paper is KAES contribution (10-106-J).

+ Corresponding author:mortigao@ksu.edu

Received 19 October 2009

Accepted 17 December 2009 or the vector in order to reduce pathogen transmission from infected to uninfected hosts. Such molecules (i.e., antigens) may be inoculated into the vertebrate host as purified proteins inducing the host immune system to produce specific antibodies (Singh \& O’Hagan 1999). Alternatively, antibodies can be raised by inoculating the host with recombinant DNA plasmids containing the gene encoding such molecules (Lobo et al. 1999, Coban et al. 2004, Kongkasuriyachai et al. 2004, LeBlanc et al. 2008). The expression and secretion of the specific protein into host tissues induce the immune system to produce antibodies against such proteins (Abdulhaqq \& Weiner 2008). To boost the immune response of the vertebrate, antigens are usually inoculated in conjunction with adjuvants. Nevertheless, the mechanisms by which adjuvants improve the immune response are still poorly understood (Singh \& O'Hagan 1999, Aguilar \& Rodriguez 2007). The specific antibodies produced against pathogen and/or vector antigens will interfere with the development of the pathogen within the vector following a blood meal on a vaccinated and infected individual.

For a molecule to be an effective TBV candidate certain basic principles must be followed. First, it has to induce high antibody titers in order to block completely pathogen development within the insect (Kubler-Kielb et al. 2007). Additionally, in case the TBV candidate is presented in an antigen/adjuvant combination, this combination has to be safe enough to the vertebrate host in order to prevent significant side effects following immunization (Saul et al. 2007, Wu et al. 2008). Ideally, a TBV candidate antigen will display low levels of polymorphisms (in field isolates) so that a unique antigen may be used to produce a TBV capable of recognizing all the field variants of that specific antigen (Kocken et 
al. 1995, Drakeley et al. 1996, Duffy \& Kaslow 1997, Sattabongkot et al. 2003). Alternatively, an effective TBV may need to combine different antigens because the combined action of the antibodies against such antigens may produce a more efficient transmission-blocking result (Duffy \& Kaslow 1997, Gozar et al. 1998, Kongkasuriyachai et al. 2004).

In light of the abundance of data regarding TBVs targeting human parasites, we felt necessary to restrict the discussion on TBVs associated within this subject. Salivary antigen-based vaccines (Valenzuela 2004, Oliveira et al. 2009) that in spite of the conferred protection do not prevent transmission were not included in this review. Moreover, insecticidal vaccines (Foy et al. 2003) are only briefly mentioned as per their potential to reduce vectorial capacity (Billingsley et al. 2006). These subjects were the recent focus of many review articles (Willadsen 2004, Billingsley et al. 2006, 2008, Titus et al. 2006, Dinglasan \& Jacobs-Lorena 2008, Oliveira et al. 2009). In this review, regarding disease vectors, we limited our discussion to insect-based TBVs and to those results based on clearly identified target molecule(s).

$T B V_{s}$ parasite antigen-based TBVS - Most of the studies on TBVs to date were conducted using antibodies targeting antigens expressed on the surface of sexual stage of malaria parasites (Figure, Table I). Plasmodium falciparum proteins Pfs25, Pfs28, Pfs48/45 and Pfs 230 and their orthologs in Plasmodium vivax were tested in transmission-blocking assays (Quakyi et al. 1987, Kaslow et al. 1988, Duffy \& Kaslow 1997, Hisaeda et al. 2000, Sattabongkot et al. 2003, Malkin et al. 2005, Outchkourov et al. 2008). Following is a discussion of each one of these Plasmodium-derived TBV molecules, different expression systems utilized to produce them and combination with different adjuvants.

P. falciparum-derived TBV candidate - Pfs25 $\mathrm{Pfs} 25$ is a $25 \mathrm{kDa}$ protein expressed on the surface of zygote and ookinete stages of $P$. falciparum and consists of four tandem epidermal growth factor (EGF) domains (Kaslow et al. 1988). The TBV potential of Pfs 25 was demonstrated using the Vaccinia virus as delivery systems of this antigen to mammalian hosts (Kaslow et al. 1991) or using recombinant Pfs25 expressed in yeast (Barr et al. 1991, Kaslow et al. 1991). With the Vaccinia virus system, infectivity of Anopheles freeborni with $P$. falciparum following an infection on artificial blood meal was reduced to $40 \%$ when $25 \mu \mathrm{g} / \mathrm{mL}$ of Pfs 25 monoclonal antibodies was added to the blood meal. Infectivity was almost eliminated when the concentration of monoclonal antibodies was increased to $200 \mu \mathrm{g} / \mathrm{mL}$ and transmission blocking activity was complete when polyclonal antibodies were used (Kaslow et al. 1991). A recombinant Pfs25 expressed in yeast (Pfs25-B) also exhibited blocking activity. Sera from mice injected with Pfs-25-B in the presence of adjuvants, such as Freund's or muramyl tripeptide (MTP-MF59), reduced P. falciparum infection of A. freeborni to less than one percent of the mosquitoes tested. Likewise, mosquitoes fed on blood of Aotus trivigatus monkeys infected with $P$. falciparum

\section{TBVs}

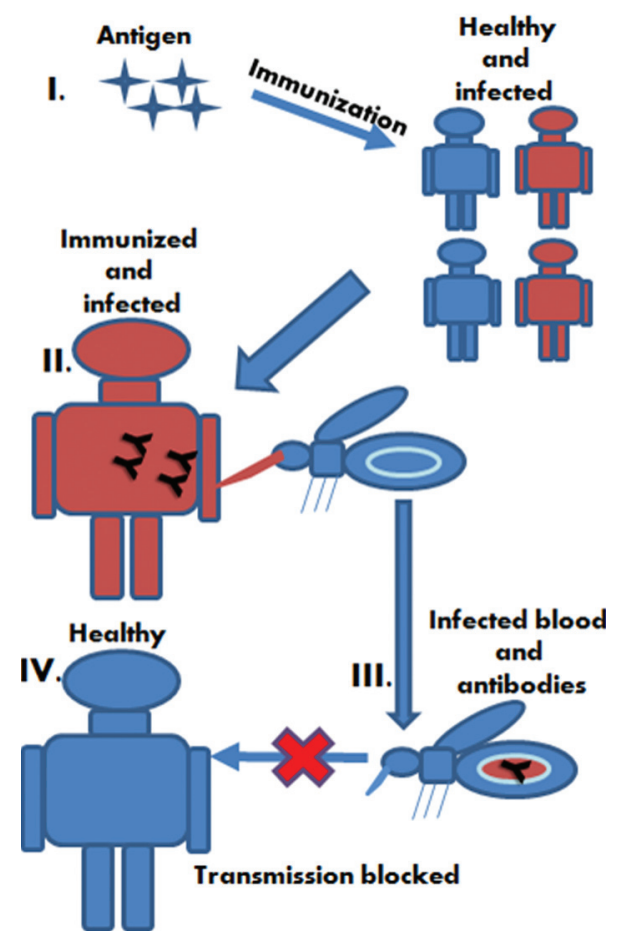

Transmission blocking vaccines (TBVs). TBV is a strategy designed to prevent transmission of a pathogen by the bite of its infected, natural vector. Frequently, TBVs rely on generating antibodies against vector molecules that are involved in pathogen development. I: healthy (blue) and infected (red) individuals are immunized with a TBV antigen; II: insect-vectors take an infected blood meal containing TBV antigen-specific antibodies; III: specific antibodies produced against the antigen inhibit pathogens development within the insect vector: IV: preventing transmission to uninfected host(s).

and immunized with Pfs25-B in combination with MTP-MF59 adjuvant exhibited infectivity of less than 10\% (Barr et al. 1991).

As a monomer, Pfs 25 was shown to be poorly immunogenic (Kubler-Kielb et al. 2007) and different forms of this protein, including various Pfs 25 dimers (obtained from single or two-step linkage conjugations) or Pfs 25 linked to immunogenic domains (such as OVA and the Pseudomonas aeruginosa recombinant exoprotein A), were tested for higher induction of antibody titers. Two of the Pfs 25 dimers obtained via two-step conjugation of amide linkages displayed the greatest immunogenicity, which was further increased when injected with alum (Kubler-Kielb et al. 2007).

Nasal immunization with Pfs 25 in the murine model also was tested (Arakawa et al. 2005). Pfs 25 expressed in yeast (Pichia pastoris) was administered intranasally with a cholera toxin adjuvant in mice. This route of immunization induced both mucosal and systemic antibodies that were capable of eliminating $P$. falciparum oocyst emergence in Anopheles dirus (Table I) (Arakawa et al. 2005).

A phase I (human safety assessment) trial of $P$. pastoris-expressed Pfs 25 antigen using Montanide ISA 


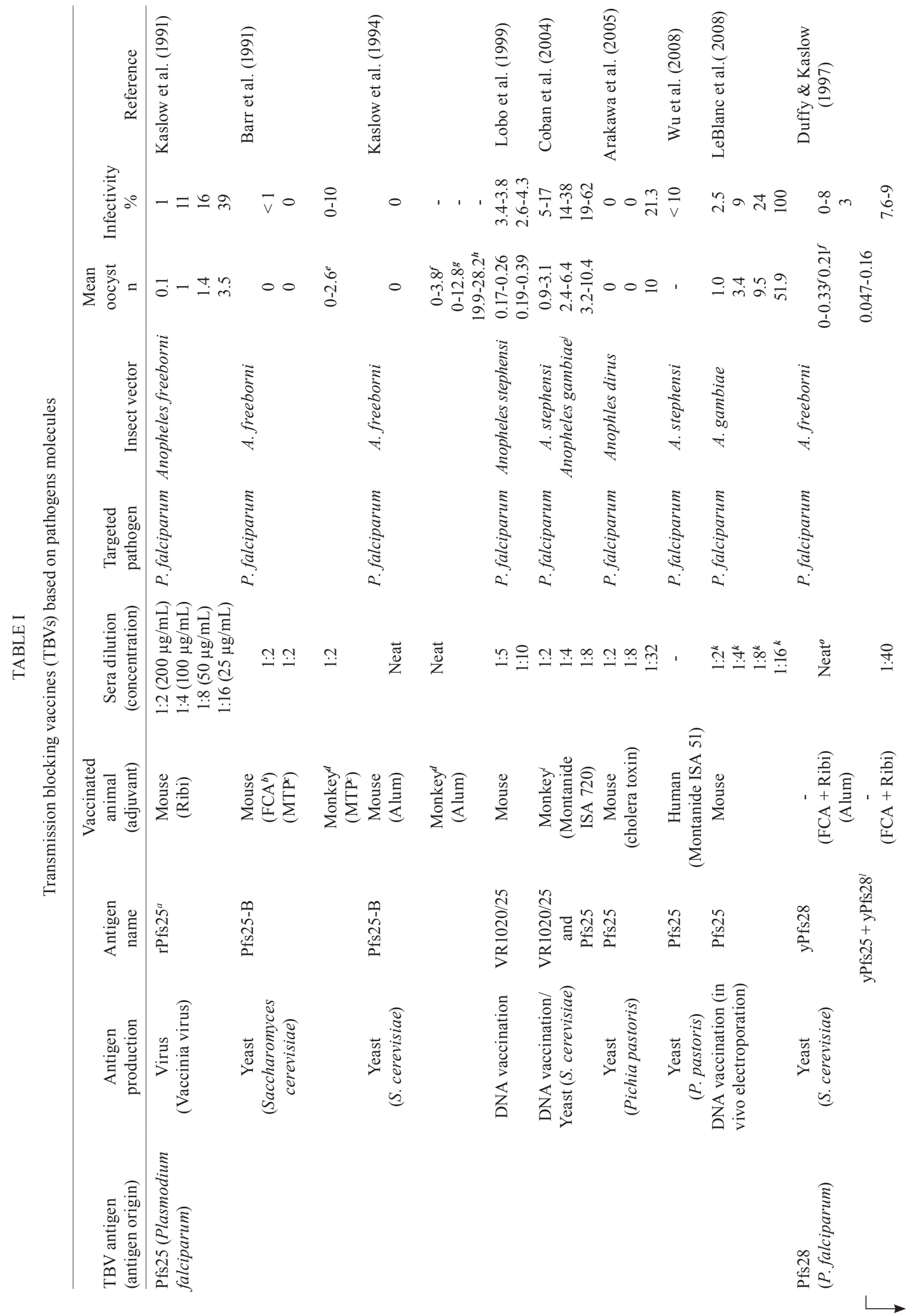




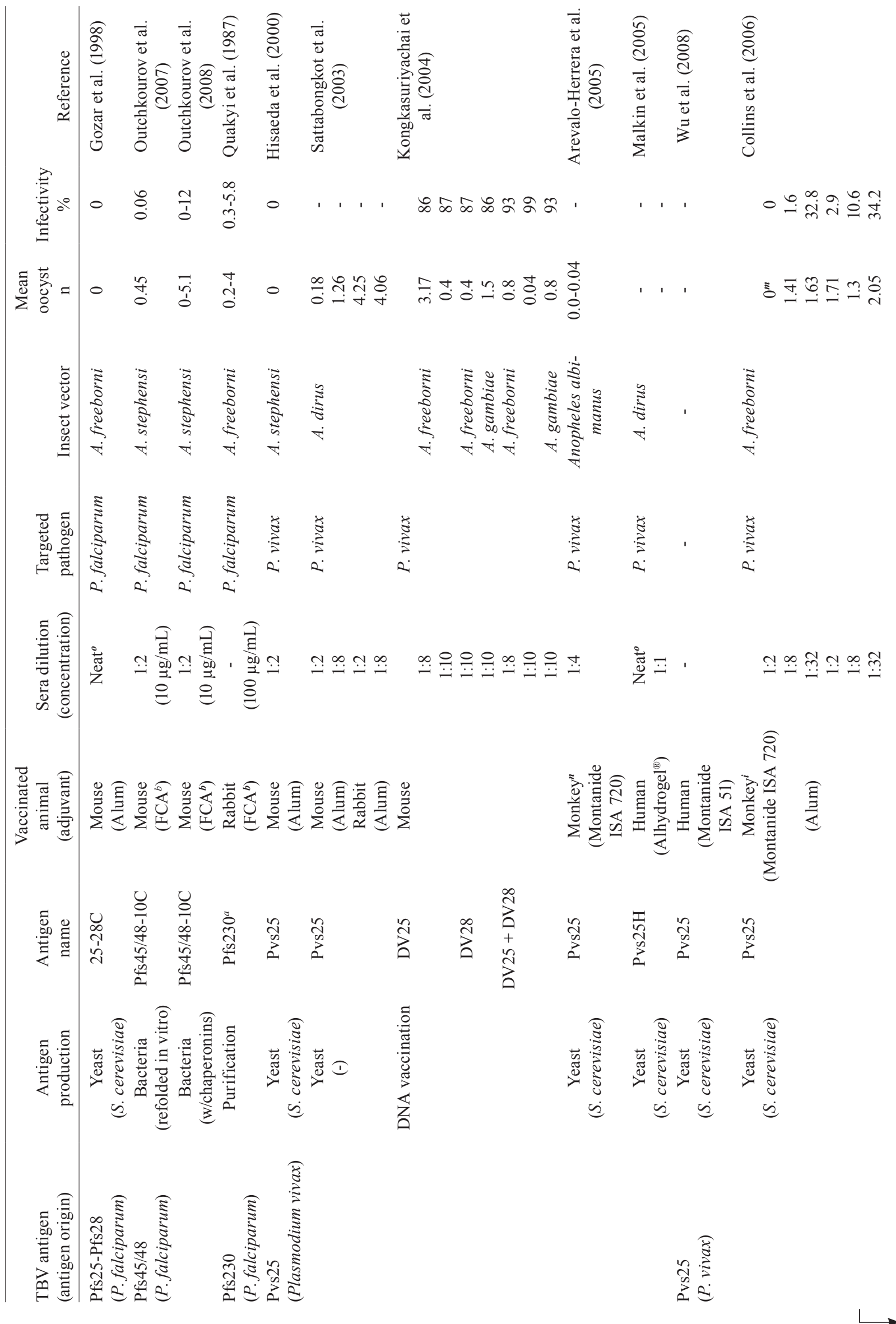




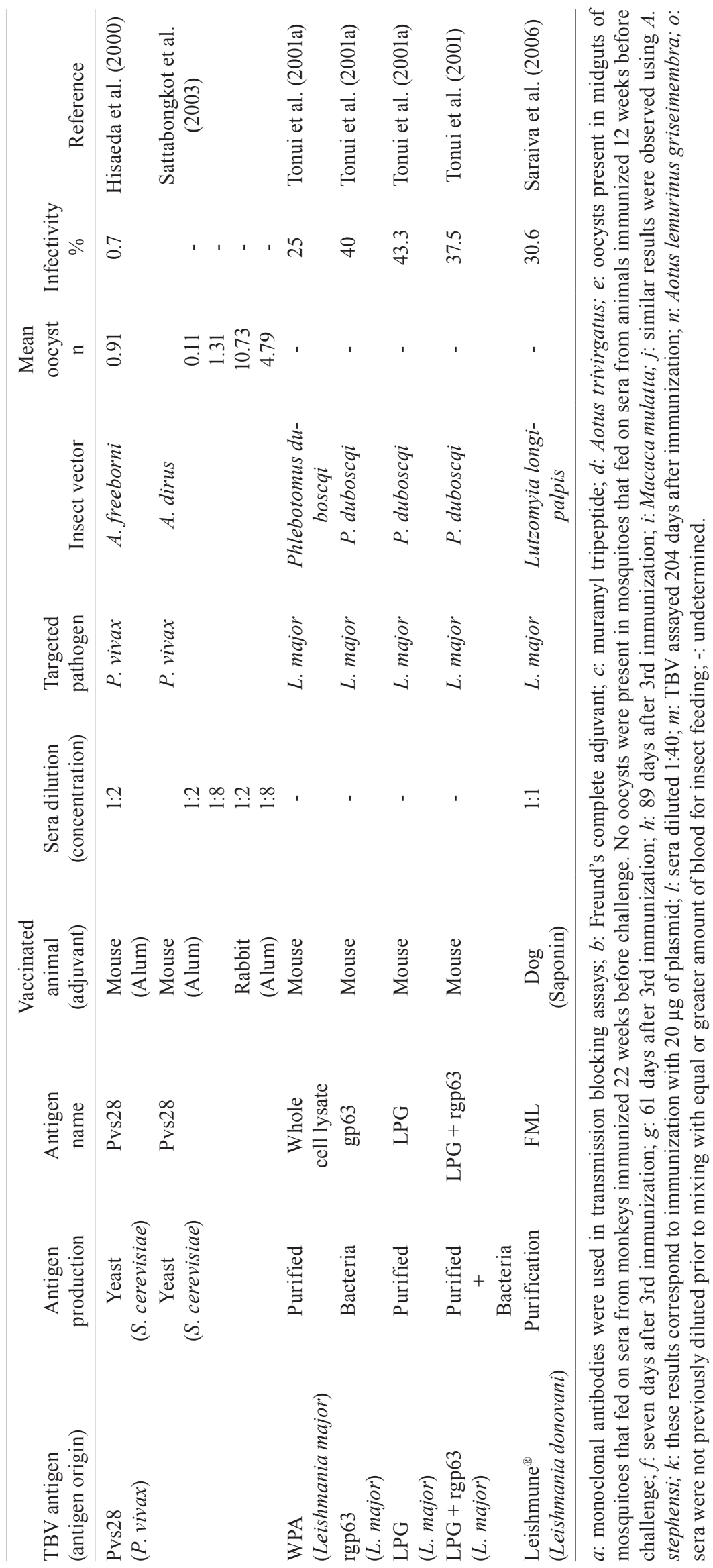


51 adjuvant was carried out (Wu et al. 2008). Although anti-Pfs 25 human serum inhibited P. falciparum oocyst intensity in Anopheles stephensi by more than 90\%, reactogenicity (local and systemic) in human volunteers prevented Montanide ISA 51 to be used as an adjuvant with Pfs25 (Wu et al. 2008).

TBV potential of DNA-based Pfs 25 vaccines also have been assessed in murine (Lobo et al. 1999) and non-human primate models (Coban et al. 2004). For both animal models, vaccination was carried out using plasmids encoding Pfs 25 or Pfg27, or a combination of plasmids expressing either one of these proteins, or expressing a chimeric Pfs25-Pfg27 protein. Pfg27 antigen is mainly expressed on the surface of $P$. falciparum gametocytes (Lobo et al. 1999, Coban et al. 2004). In the mouse model, DNA immunization with the plasmids encoding Pfs 25 alone or in combination with Pfg27-encoding plasmids induced high antibody titers after only two inoculations without adjuvants (Lobo et al. 1999). Injection of plasmids encoding Pfs 25 alone displayed the greatest TBV activity (oocyst infectivity reduction: 96.2-96.6\%), followed by co-immunization with plasmids encoding Pfs 25 or Pfg27 (oocyst infectivity reduction: $94.8-96.4 \%$ ). Immunization with plasmids expressing Pfg27 alone did not induce effective TBV potential (Lobo et al. 1999). In contrast, immunization of rhesus monkeys (Macaca mulatta) with plasmids expressing Pfs 25 or a chimeric Pfs 25 -Pfg27 protein did not stimulate the production of high antibody titers, even after four inoculations and was incapable of inhibiting P. falciparum development in A. stephensi (Coban et al. 2004). However, subsequent single immunization with the yeast-expressed $\mathrm{Pfs} 25$ protein, along with Montanide ISA 720 adjuvant, significantly reduced $P$. falciparum oocyst numbers by up to $95 \%$ in A. stephensi (Coban et al. 2004). Heterologous boosting with recombinant Pfs25 did not induce TBV activity in the immunization with plasmids expressing the chimeric Pfs25-Pfg27 (Coban et al. 2004). Therefore, heterologous boosting of Pfs 25 DNA-based TBV vaccine with a recombinant protein plus adjuvant seems to be a reliable option for a phase 1 trial as this vaccination strategy induced strong reduction of $P$. falciparum development in mosquitoes as well as was unable to affect the health of non-human primates (Coban et al. 2004).

An attempt to boost the immunogenicity of a Pfs25based DNA vaccine was performed via in vivo electroporation (LeBlanc et al. 2008). This method elicited similar anti-Pfs 25 antibody titers in mice as immunization carried out with 100 fold more plasmid without electroporation. Additionally, membrane feeding assays using either serum, derived from Pfs25-DNA plasmid immunization after electroporation or from Pfs25-DNA plasmid (100 fold concentrated) immunization without electroporation, induced similar P. falciparum oocyst intensity reduction in Anopheles gambiae (approximately $85 \%$ ). In vivo electroporation of Pfs- 25 encoding plasmids could be tested to boost the immune response of TBVs in non-human primates and eventually in phase 1 trials (LeBlanc et al. 2008).
P. falciparum-derived TBV candidate - Pfs 28 - Pfs 28 is a $28 \mathrm{kDa} P$. falciparum conserved protein expressed on the surface of retorts, a transitional stage between zygote and ookinete. This antigen also was tested in transmission blocking activity assays. Antibodies produced by the injection of yeast-expressed Pfs 28 (yPfs28), in the presence of alum, significantly reduced the infectivity of A. freeborni mosquitoes with P. falciparum. Lower infectivity was exhibited when vaccination was carried out with yPfs 28 and yPfs 25 antigens injected together (Duffy \& Kaslow 1997).

Transmission blocking activity against $P$. falciparum was further improved when Pfs 25 and Pfs 28 were expressed as a unique chimeric protein in yeast, the $25-28 \mathrm{c}$ recombinant protein. Vaccination with the 25-28c recombinant protein led to complete arrest of oocyst development earlier, using a lower dose and for a greater amount of time, than vaccination with either Pfs 25 or Pfs 28 alone or a combination of both (Gozar et al. 1998).

P. falciparum-derived TBV candidate - Pfs $48 / 45$ Another TBV candidate to control spread of $P$. falciparum is $\mathrm{Pfs} 48 / 45$. The $P f s 48 / 45$ gene encodes a unique protein that migrates as a double band under non-reducing conditions (Milek et al. 2000). This protein is expressed on $P$. falciparum gametocyte and gamete surfaces and has a central role in male gamete fertility (van Dijk et al. 2001). Attempts to express this protein in its native form using different systems, such as bacteria (Milek et al. 1998a), Vaccinia virus (Milek et al. 1998b) and yeast (Milek et al. 2000) have failed due to improper folding. However, expression of a truncated form of Pfs48/45 (Pfs48/45-10C) in E. coli followed by steps of chemical-induced refolding produced $10-20 \%$ of correctly folded Pfs48/45-10C (Outchkourov et al. 2007). Mice polyclonal antibodies produced against Pfs48/45-10C recombinant protein (in the presence of Freund's adjuvant) reduced the intensity of $P$. falciparum oocysts in A. stephensi by more than 15 fold (Outchkourov et al. 2007). Additionally, antibody titers were sufficient to induce transmission blocking effects (Outchkourov et al. 2007). Much improved refolding of the truncated Pfs $48 / 45-10 \mathrm{C}$ was obtained through co-expression of $E$. coli chaperones (i.e., $90 \%$ of the recombinant protein was properly folded and stable) (Outchkourov et al. 2008). Immunization of mice with this recombinant protein led to production of antibody titers that were capable of reducing $P$. falciparum oocyst intensity in $A$. stephensi by at least $88 \%$ in 11 out of 12 assays (Outchkourov et al. 2008).

Furthermore, with regards to the application of Pfs $48 / 45$ as a potential TBV against malaria, the variability of Pfs $48 / 45$ from culture and field isolates from many countries was analyzed (Kocken et al. 1995, Drakeley et al. 1996). The results obtained indicated low levels of polymorphism in the overall gene among either in vitro cultures or field isolates (Kocken et al. 1995, Drakeley et al. 1996).

P. falciparum-derived TBV candidate - Pfs 230 - Another $P$. falciparum protein tested in TBV assays was Pfs230, a $230 \mathrm{kDa}$ protein expressed on the surface 
of gametocytes. Although antibodies against Pfs230 blocked the development of $P$. falciparum in the midguts of $A$. freeborni, the transmission blocking activity of antiPfs230 monoclonal antibodies was completely lost when complement was inactivated. Thus, the blocking activity of anti-Pfs-230 antibodies was detected only when complement proteins were present (Quakyi et al. 1987).

P. vivax-derived TBV candidates - Pvs25 AND Pvs28 - P. vivax sexual stage surface proteins, orthologs of $P$. falciparum TBV candidates, also have been isolated and tested in transmission blocking experiments. Pvs25, a Pfs 25 ortholog, is expressed on the surfaces of the insect-stages, zygotes and mature ookinetes, whereas Pvs28, a Pfs28 ortholog, is mainly expressed on retorts and mature ookinetes (Hisaeda et al. 2000).

Transmission blocking experiments using antibodies against either Pvs25 or Pvs28 were tested (Hisaeda et al. 2000). Four species of mosquitoes were artificially fed on a mixture of $P$. vivax-infected chimpanzee blood in the presence of antibodies (raised in mice co-injected with alum). $P$. vivax ookinete development was completely blocked by the anti-serum against Pvs25 (Hisaeda et al. 2000). Vaccination against Pvs25 and Pvs28 also presented efficient transmission blocking activity against $P$. vivax isolated from human patients, despite polymorphism in these proteins (Sattabongkot et al. 2003).

Sera of Aotus lemurinus griseimembra monkeys immunized with Pvs25 and Montanide ISA 720 adjuvant also were used in a TBV assay (Arevalo-Herrera et al. 2005). These sera were capable of blocking human-derived $P$. vivax oocyst intensity in Anopheles albimanus by more than $98 \%$, reaching complete oocyst inhibition in most of the replicates (Arevalo-Herrera et al. 2005).

Similar to the transmission blocking activity of the Aotus anti-Pvs25 sera observed previously (ArevaloHerrera et al. 2005), TBV activity of anti-Pvs25 sera raised in rhesus monkeys also was assessed (Collins et al. 2006). The boosting effect of two adjuvants (alum and Montanide ISA 720) was compared. Immunization with a combination Pvs25 and Montanide ISA 720 induced a stronger antibody response than Pvs25 and alum in rhesus monkeys, reaching 100\% reduction of oocyst intensity in A. freeborni, 204 days after immunization (Collins et al. 2006).

Transmission blocking activity of Pvs 25 has been evaluated in phase 1 human trials. Antibodies to Pvs25 were raised in healthy human volunteers using Alhydrogel $^{\circledR}$ (aluminium hydroxide gel) as adjuvant and the sera was mixed with the $P$. vivax-infected blood taken from infected patients from an endemic region. This mixture of human infected blood and human anti-Pvs25 serum was used to artificially feed $A$. dirus. The results from the study revealed significant interference in $P$. vivax development within mosquito midgut caused by the human anti-Pvs25 sera. Additionally, long lasting antibody titers were elevated and no reactogenicity (side effects) was observed (Malkin et al. 2005). Nevertheless, higher antibody titers are necessary for successful control of $P$. falciparum transmission by mosquitoes in endemic areas (Malkin et al. 2005). A second phase 1 trial, also using Pvs25 as a potential TBV was carried out using Montanide ISA 51 as an adjuvant. Due to induced local and systemic reactions in the vacinees, use of this antigen-adjuvant combination in humans was halted (Wu et al. 2008).

Immunogenicity of Pvs25 and Pvs28 DNA-based TBVs was also assessed in a murine model (Kongkasuriyachai et al. 2004). Many immunization schemes, including Pvs 25 or Pvs 28 plasmids alone, in combination, boosted with the recombinant Pvs 25 or Pvs 28 proteins plus aluminium hydroxide adjuvant or a combination of plasmids encoding Pvs25 and Pfs25, were carried out. Overall, all these schemes efficiently elicited comparable levels of antibodies and inhibited $P$. vivax development in $A$. freeborni and $A$. gambiae (at least $74 \%$ oocyst intensity reduction) (Kongkasuriyachai et al. 2004).

Other pathogen molecule-based TBV candidates In regard to proteins expressed on the surface of parasites (other than Plasmodium) transmitted to humans by insect vectors (Table I) only a limited number has been tested as potential TBVs (Tonui et al. 2001a, b, Saraiva et al. 2006).

In Leishmania major, the two most abundant surface antigens, lipophosphoglycan (LPG) and gp63, were tested as TBVs. Phlebotomus duboscqi sand flies were partially fed on mice immunized with purified native LPG, recombinant gp63 (rgp63) expressed in bacteria, crude L. major lysate (WPA), or a cocktail of LPG and rgp63. The sand flies were subsequently fed on L. major-infected mice. The results indicated that serum against WPA and the two protein-cocktail exhibited greater L. major blocking activity than sera against either LPG or gp63 (Tonui et al. 2001a). However, blocking of L. major development was due to damage of the midgut epithelial layer, probably caused by immune-active substances present in the blood of the pre-vaccinated mice (Tonui et al. 2001a).

Interestingly, a commercially available vaccine for canine visceral leishmaniasis (Leishmune ${ }^{\circledR}$ ) was recently shown to function as a TBV in sand flies (Saraiva et al. 2006). Leishmune ${ }^{\circledR}$ [surface molecule (FML) -vaccine] is a protective vaccine made of Leishmania donovani fucose-mannose ligand and the adjuvant saponin, which was successfully tested in a phase III vaccine trial (da Silva et al. 2000). Although the FML was isolated from L. donovani, Leishmune ${ }^{\circledR}$ exhibited transmission blocking activity in the New World sand fly Lutzomyia longipalpis when infected with Leishmania infantum chagasi (Saraiva et al. 2006). Antibodies produced in dogs following Leishmune ${ }^{\circledR}$ injection reduced $\mathrm{Lu}$. longipalpis infectivity by $79.3 \%$ and parasite load by $74.3 \%$ even after 12 months of immunization (Saraiva et al. 2006).

Insect-based TBVS - Proteins expressed within insect vector tissues and that may interact with pathogens also have been tested as TBV candidates (Table II). Insectbased TBV candidates include (structural) proteins that are expressed by the insect midgut (Lal et al. 2001), midgut enzymes that play a role in blood digestion (Lavazec et al. 2007) and parasite receptors expressed by the epithelial cells lining the midgut (Kamhawi et al. 2004, Dinglasan et al. 2007). 


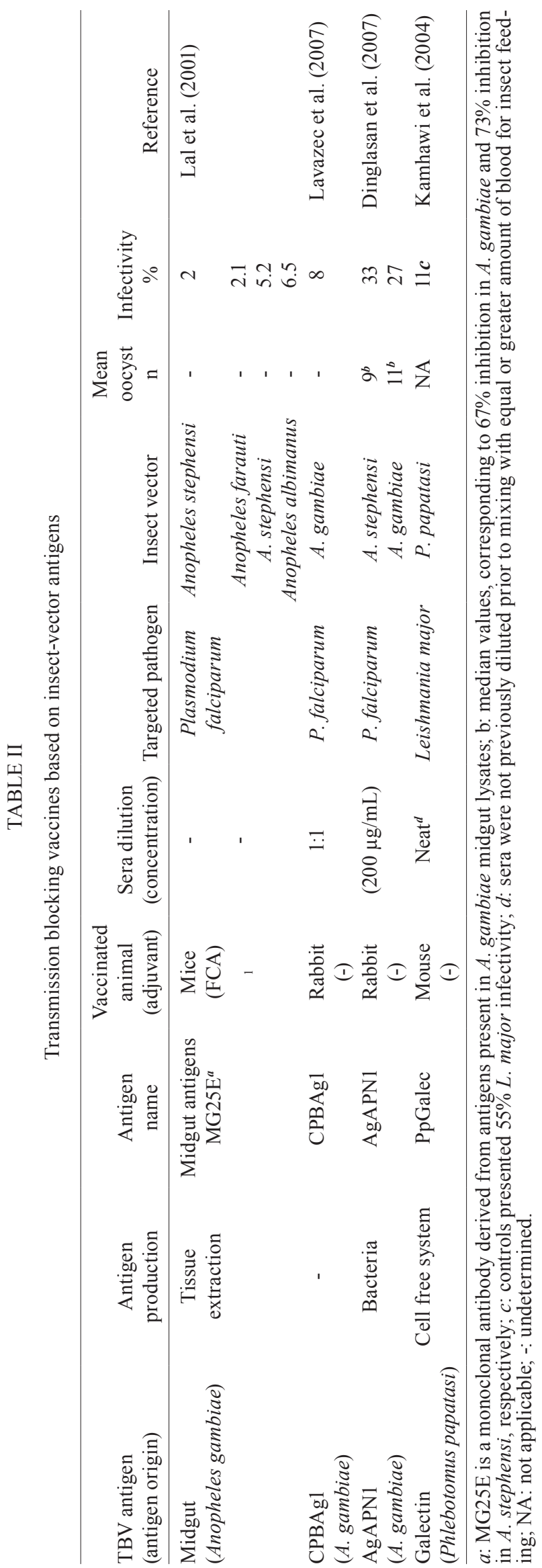

In mosquitoes, polyclonal antibodies against $\mathrm{A}$. gambiae midgut proteins nearly completely reduced the intensity of $P$. falciparum oocysts $(98 \%)$ and sporozoites (96\%) within A. stephensi tissues. Also, A. gambiaederived anti-midgut monoclonal antibodies-inhibited development of $P$. falciparum and $P$. vivax in different Anopheles species (Lal et al. 2001). Additionally, these antibodies also can be used to reduce insect vector densities (vector-blocking vaccines) because they reduce vector survivorship and fecundity (Lal et al. 2001). Antibodies against carboxypeptidase cpbAg1 from $A$. gambiae reduced $P$. falciparum infectivity by more than $92 \%$ seven days after an infectious artificial blood feeding (Lavazec et al. 2007). In addition to the effect on the number of oocysts per infected mosquito, anti-cpbAg1 strongly reduced mosquito progeny (Lavazec et al. 2007). Antibodies to a midgut aminopeptidase (AgAPN1), which is one of the $P$. falciparum receptors in the A. gambiae midgut, were used to reduce $P$. falciparum oocyst intensity in $A$. gambiae and A. stephensi by $73 \%$ and $67 \%$, respectively (Dinglasan et al. 2007).

Sand fly-based TBV have also been suggested as a potential strategy against Leishmania transmission, especially in those situations where the disease displays an anthroponotic transmission profile, or where domestic dogs play a critical role as reservoirs of the parasite. The utilization of sand fly molecules as TBVs was contemplated following the characterization of PpGalec, a galectin (galactose-binding) -like protein. PpGalec is a expressed on the surface of the midgut cells in Phlebotomus papatasi and serve as a receptor for $L$. major LPG attachment (Kamhawi et al. 2004). Interestingly, P. papatasi fed artificially on mouse blood containing $L$. major amastigotes and mixed with sera from PpGalec-immunized mice displayed a reduction of $86 \%$ on the levels of L. major infection within the sand fly midgut. Moreover, no infectious metacyclic forms were detected from the flies fed on anti-PpGalec sera (Kamhawi et al. 2004).

Although this study clearly demonstrated that by using anti-sera it is possible to block a parasite receptor within the midgut of its vector, the issue here is that $\mathrm{Pp}$ Galec is present within a vector that is associated with zoonotic disease. To succeed against the transmission of a human parasite, a TBV molecule has to function in vector-parasite pairs that are meaningful to human disease.

In a similar approach, anti-sera raised against another sand fly molecule, PpChit1, also characterized from P. papatasi (subgenus Phlebotomus) inhibited the chitinolytic activity of the midgut of other sand fly species in vitro, including $P$. duboscqi (also subgenus Phlebotomus), but even from a sand fly (Phlebotomus argentipes) of a distant subgenus (Euphlebotomus) (Ramalho-Ortigao et al. 2005). These data suggested a significant cross-species activity, which is what is at stake here. Thus, using a model disease vector such as $P$. papatasi for investigations related to the identification of candidate TBVs will provide significant clues to molecules that display a broad spectrum of activity, have counterparts (orthologs or paralogs) in other sand fly vectors and are potentially directly associated with the development of Leishmania. Thus, when such molecules are targeted, via antibodies 
or other strategies, it will lead to the disruption of the development of the parasite and its transmission to humans and domestic animals.

Challenges and perspectives on TBVS - In addition to identifying TBV candidates that are effective and may span different insect vector species, challenges to TBV development also encompass antigenic variability present in field isolates (Kocken et al. 1995, Drakeley et al. 1996, Duffy \& Kaslow 1997, Sattabongkot et al. 2003), immunogenicity of such antigens (Kubler-Kielb et al. 2007), reactogenicity caused by adjuvants (Saul et al. 2007, Wu et al. 2008), non-specific responses (Quakyi et al. 1987, Tonui et al. 2001a) and improper folding of antigens (Kaslow et al. 1994, Milek et al. 1998a, b, 2000). Natural antigenic boosting is another important issue that must be dealt with (Arevalo-Herrera et al. 2005).

Antigens expressed on the surface of insect-stage parasites have been postulated as TBV candidates because they seem not to be under the selective pressure mediated by the vertebrate immune system. Consequently, these proteins could exhibit low levels of polymorphisms, favoring the efficacy of vaccines derived from a unique antigenic variant (Sattabongkot et al. 2003). Even though Pvs25, Pvs28 and Pfs48/45 proteins from different Plasmodium field isolates are polymorphic antigens, the amount of variation of these antigens does not preclude their use as TBVs (Drakeley et al. 1996, Duffy \& Kaslow 1997, Sattabongkot et al. 2003). On the other hand, antigenic variability of vector TBV candidates has not yet been assessed.

Another challenge to the development of TBVs is related to the strength of the immune response mediated by the TBV candidate. For example, Pfs 25 does not stimulate a strong immune response to generate antibody titers that could reduce parasite development efficiently in mosquito field populations (Kubler-Kielb et al. 2007), nor does Pvs25 administered with Alhydrogel ${ }^{\circledR}$ (Malkin et al. 2005). In order to booster the immunogenicity of Pvs25, a different adjuvant (Montanide ISA 51) has been tested (Wu et al. 2008). Similarly, immunogenicity of Pfs 25 has been improved by modifications of the $3 \mathrm{D}$-structure of this protein (Kubler-Kielb et al. 2007) as well as by the use of different adjuvants (Barr et al. 1991, Kaslow et al. 1994).

An alternate strategy to boost the immune response of TBV antigens was assessed through DNA-based TBV immunization (Lobo et al. 1999, Coban et al. 2004, Kongkasuriyachai et al. 2004, LeBlanc et al. 2008). Besides the lower cost and stability of these vaccines (LeBlanc et al. 2008), DNA-based TBVs alone or boosted with recombinant proteins elicit high antibody titers (Lobo et al. 1999, Coban et al. 2004, Kongkasuriyachai et al. 2004). These vaccines were used to block transmission of malaria parasites and have demonstrated safety when used in non-human primates (Lobo et al. 1999, Coban et al. 2004, Kongkasuriyachai et al. 2004). Electroporation of DNA-based TBVs enhanced antibody production in mice and also are considered an alternative to boosting antibody response to DNA vaccines in larger animals (LeBlanc et al. 2008).
The use of adjuvants to boost TBV antigen response is a significant issue to be resolved, as certain types of adjuvants induce reactogenicity in humans (Wu et al. 2008) and animal models (Saul et al. 2007). In humans, a combination of Pvs25 and Alhydrogel ${ }^{\circledR}$ was shown to be safe in a phase 1 trail (Malkin et al. 2005). In contrast, Pvs25 and Montanide ISA 51 caused local and systemic adverse effects (Wu et al. 2008). In rhesus monkeys, while Pvs25$\mathrm{H}$ Montanide ISA 720 immunization induced higher levels (10 fold) of antibodies than the Pvs25-Alhydrogel ${ }^{\circledR}$ (aluminium hydroxide gel) combination, it also led to local reactogenicity (Saul et al. 2007). In a subsequent experiment, the immunization of monkeys with similar or lower doses of Pfs25/Montanide ISA 720 did not induce local reactogenicity or stimulated antibody levels following a second round of injection with a dose as high as that in the first experiment (Saul et al. 2007). A qualitative difference in immune response between the first and second boosting may be responsible for the reactogenic reactions caused by Pfs25/Montanide ISA 720. As suggested by Saul et al. (2007), this may be prevented by an immunization scheme using a small priming dose followed by a second dose several months later (Saul et al. 2007). Therefore, combinations of TBV candidate antigens and adjuvants need to be thoroughly tested in order to define the specific dose to be administered and using the safest antigen-adjuvant combination.

Another interesting aspect of TBVs is the possibility of natural boosting of the immune response of animals infected with a pathogen (i.e., pre-immunized) (Milek et al. 1998a, b, Arevalo-Herrera et al. 2005). Hence, candidate TBV proteins expressed on the surface of both insect-stage and blood-stage pathogens may induce activation of the immune response in infected hosts vaccinated with the same antigens (Arevalo-Herrera et al. 2005). However, this approach may not be suitable to every TBV, such as Pvs25 which displays low expression in blood-stage $P$. vivax (Arevalo-Herrera et al. 2005) and has yet to be demonstrated for the Plasmodium TBVantigen candidates that are expressed during gametocytogenesis, for example, Pfs230 (Quakyi et al. 1987) and Pfs48/45 (Milek et al. 1998a, b).

Proper folding of the TBV candidate protein following expression via recombinant techniques also may affect the efficacy of the vaccinating antigen. As indicated above, expression of Pfs 25 in prokaryotic systems resulted in improper folded proteins (Kaslow et al. 1994), which was solved by expression in yeast (Kaslow et al. 1994) or in Vaccinia virus-infected mammalian cells (Kaslow et al. 1991). Conversely, Pfs48/45 expressed in bacteria (Milek et al. 1998a), Vaccinia virus-infected mammalian cells (Milek et al. 1998b), or yeast (Milek et al. 2000) resulted in the production of denatured antigens, improper for host immunization. Properly folded Pfs48/45 was recently obtained by chemically induced re-folding of an E. coli-expressed truncated Pfs $48 / 45$ (Outchkourov et al. 2007) and through co-expression of this antigen with E. coli chaperons (Outchkourov et al. 2008). Thus, the system of choice for recombinant expression can significantly affect the outcome of the TBV candidate. 
Future directions on TBV research - The low antibody-responses induced by immunization with Plasmodium-surface proteins as well as the reactogenicity caused by some adjuvants seem to be the main constraints on the development of anti-malaria TBVs (Kubler-Kielb et al. 2007, Saul et al. 2007, Wu et al. 2008). Although antigen engineering has been carried out in order to improve antibody-response (Kubler-Kielb et al. 2007) and some immunization routines appear to reduce side effects (Saul et al. 2007), human-safe adjuvants that induce strong antibody responses still need to be developed for TBV antigens to induce sufficient antibody titers to reduce transmission in endemic areas. With regards to other pathogens besides malaria parasites, assessment of potential TBV candidate antigens has yet to be undertaken.

In regards to insect-based TBV candidate molecules, the number of TBV antigens available is still reduced and needs to be extended to target other vector species. In addition to assessing a TBV candidate molecule that prevents pathogen development within insect vector tissues, an effect on the vector survivorship is also one of the main objectives.

Reduction of vector survival is thought to interfere exponentially with vectorial capacity (Black \& Moore 2004, Billingsley et al. 2006, 2008), as the time available for pathogen development within the vector is significantly shortened. Despite several studies showing that insect feeding on blood of animals immunized with insect tissue homogenates exhibit reduced survivorship, most of these studies suffered from high experimental variability (Billingsley et al. 2006). However, one study has shown that immunization with a unique insect molecule (mucin) can induce an immune response capable of killing insect vectors via a cell-mediated response (Foy et al. 2003). Thus, an ideal TBV antigen should reduce parasite development, reducing vector competence (a linear parameter in the vectorial capacity equation), as well as vector survivorship (the exponential parameter). These two effects associated can lead to thorough reduction of vectorial capacity and disease burden in endemic areas.

TBV could also be able to reduce survivorship of different species of insect vectors, via immunization with conserved antigens, as proposed by Canales et al. (2009), providing protection to pathogens transmitted by different vectors. However, significant cross-species effects have yet to be demonstrated.

Significant funding and research efforts are currently been invested to develop novel mechanisms to control insect-borne diseases. Among the various strategies investigated, TBVs may soon be applied towards the control of vector-borne diseases, especially malaria and leishmaniasis. This assumption is supported by at least two recent developments, the first being the approval of the P. vivax Pvs25 antigen as TBV during a phase I human trial (Malkin et al. 2005, Saraiva et al. 2006). Another is the use of the fucose-mannose ligand from $L$. donovani as a vaccine against canine visceral leishmaniasis following a phase III trial for animal application (da Silva et al. 2000, Saraiva et al. 2006). Interestingly, Leishmune ${ }^{\mathrm{TM}}$ also function as a TBV preventing the development of $L$. infantum chagasi in its natural vector the sand fly $L u$. longipalpis (Malkin et al. 2005, Saraiva et al. 2006).

Currently, many laboratories are investigating several leads that potentially can be applied as TBV to prevent insect-transmitted pathogens. However the outcome of the research with current TBV candidates, it is clear to investigators that novel strategies are needed in order to reduce the current burden of vector-borne diseases in general.

\section{ACKNOWLEDGEMENT}

To Dr. Yoonseong Park (Kansas State University), for critically reviewing the manuscript.

\section{REFERENCES}

Abdulhaqq SA, Weiner DB 2008. DNA vaccines: developing new strategies to enhance immune responses. Immunol Res 42: $219-232$.

Aguilar JC, Rodriguez EG 2007. Vaccine adjuvants revisited. Vaccine 25: $3752-3762$.

Arakawa T, Komesu A, Otsuki H, Sattabongkot J, Udomsangpetch R, Matsumoto Y, Tsuji N, Wu Y, Torii M, Tsuboi T 2005. Nasal immunization with a malaria transmission-blocking vaccine candidate, $\mathrm{Pfs} 25$, induces complete protective immunity in mice against field isolates of Plasmodium falciparum. Infect Immun 73: $7375-7380$.

Arevalo-Herrera M, Solarte Y, Yasnot MF, Castellanos A, Rincon A, Saul A, Mu J, Long C, Miller L, Herrera S 2005. Induction of transmission-blocking immunity in Aotus monkeys by vaccination with a Plasmodium vivax clinical grade PVS25 recombinant protein. Am J Trop Med Hyg 73: 32-37.

Barr PJ, Green KM, Gibson HL, Bathurst IC, Quakyi IA, Kaslow DC 1991. Recombinant Pfs25 protein of Plasmodium falciparum elicits malaria transmission-blocking immunity in experimental animals. J Exp Med 174: 1203-1208.

Billingsley PF, Baird J, Mitchell JA, Drakeley C 2006. Immune interactions between mosquitoes and their hosts. Parasite Immunol 28: 143-153.

Billingsley PF, Foy B, Rasgon JL 2008. Mosquitocidal vaccines: a neglected addition to malaria and dengue control strategies. Trends Parasitol 24: 396-400.

Black IV WC, Moore CG 2004. Population biology as a tool to study vector-borne diseases. In WC Marquardt, Biology of disease vectors, Elservier Academic Press, Burlington, p. 187-206.

Canales M, Naranjo V, Almazan C, Molina R, Tsuruta SA, Szabo MP, Manzano-Roman R, Perez de la Lastra JM, Kocan KM, Jimenez MI, Lucientes J, Villar M, de la Fuente J 2009. Conservation and immunogenicity of the mosquito ortholog of the tick-protective antigen, subolesin. Parasitol Res 105: 97-111.

Coban C, Philipp MT, Purcell JE, Keister DB, Okulate M, Martin DS, Kumar N 2004. Induction of Plasmodium falciparum transmission-blocking antibodies in nonhuman primates by a combination of DNA and protein immunizations. Infect Immun 72: 253-259.

Collins WE, Barnwell JW, Sullivan JS, Nace D, Williams T, Bounngaseng A, Roberts J, Strobert E, McClure H, Saul A, Long CA 2006. Assessment of transmission-blocking activity of candidate Pvs25 vaccine using gametocytes from chimpanzees. Am J Trop Med Hyg 74: 215-221.

Coutinho-Abreu IV, Zhu KY, Ramalho-Ortigao M 2009. Transgenesis and paratransgenesis to control insect-borne diseases: current status and future challenges. Parasitol Int, in press. 
da Silva VO, Borja-Cabrera GP, Correia Pontes NN, de Souza EP, Luz KG, Palatnik M, Palatnik de Sousa CB 2000. A phase III trial of efficacy of the FML-vaccine against canine kala-azar in an endemic area of Brazil (São Gonçalo do Amaranto, RN). Vaccine 19: 1082-1092.

Dinglasan RR, Jacobs-Lorena M 2008. Flipping the paradigm on malaria transmission-blocking vaccines. Trends Parasitol 24: 364-370.

Dinglasan RR, Kalume DE, Kanzok SM, Ghosh AK, Muratova O, Pandey A, Jacobs-Lorena M 2007. Disruption of Plasmodium falciparum development by antibodies against a conserved mosquito midgut antigen. Proc Natl Acad Sci USA 104: 13461-13466.

Drakeley CJ, Duraisingh MT, Povoa M, Conway DJ, Targett GA, Baker DA 1996. Geographical distribution of a variant epitope of Pfs48/45, a Plasmodium falciparum transmission-blocking vaccine candidate. Mol Biochem Parasitol 81: 253-257.

Duffy PE, Kaslow DC 1997. A novel malaria protein, Pfs 28 and Pfs 25 are genetically linked and synergistic as falciparum malaria transmission-blocking vaccines. Infect Immun 65: 1109-1113.

Foy BD, Magalhaes T, Injera WE, Sutherland I, Devenport M, Thanawastien A, Ripley D, Cardenas-Freytag L, Beier JC 2003. Induction of mosquitocidal activity in mice immunized with Anopheles gambiae midgut cDNA. Infect Immun 71: 2032-2040.

Gozar MM, Price VL, Kaslow DC 1998. Saccharomyces cerevisiaesecreted fusion proteins Pfs 25 and Pfs 28 elicit potent Plasmodium falciparum transmission-blocking antibodies in mice. Infect Immun 66: 59-64.

Hill CA, Kafatos FC, Stansfield SK, Collins FH 2005. Arthropodborne diseases: vector control in the genomics era. Nat Rev Microbiol 3: 262-268.

Hisaeda H, Stowers AW, Tsuboi T, Collins WE, Sattabongkot JS, Suwanabun N, Torii M, Kaslow DC 2000. Antibodies to malaria vaccine candidates Pvs25 and Pvs28 completely block the ability of Plasmodium vivax to infect mosquitoes. Infect Immun 68: 6618-6623.

Kamhawi S, Ramalho-Ortigao M, Pham VM, Kumar S, Lawyer PG, Turco SJ, Barillas-Mury C, Sacks DL, Valenzuela JG 2004. A role for insect galectins in parasite survival. Cell 119: 329-341.

Kaslow DC, Bathurst IC, Lensen T, Ponnudurai T, Barr PJ, Keister DB 1994. Saccharomyces cerevisiae recombinant Pfs 25 adsorbed to alum elicits antibodies that block transmission of Plasmodium falciparum. Infect Immun 62: 5576-5580.

Kaslow DC, Isaacs SN, Quakyi IA, Gwadz RW, Moss B, Keister DB 1991. Induction of Plasmodium falciparum transmission-blocking antibodies by recombinant vaccinia virus. Science 252: 1310-1313.

Kaslow DC, Quakyi IA, Syin C, Raum MG, Keister DB, Coligan JE, McCutchan TF, Miller LH 1988. A vaccine candidate from the sexual stage of human malaria that contains EGF-like domains. Nature 333: 74-76.

Kocken CH, Milek RL, Lensen TH, Kaslow DC, Schoenmakers JG, Konings RN 1995. Minimal variation in the transmission-blocking vaccine candidate Pfs $48 / 45$ of the human malaria parasite Plasmodium falciparum. Mol Biochem Parasitol 69: 115-118.

Kongkasuriyachai D, Bartels-Andrews L, Stowers A, Collins WE, Sullivan J, Sattabongkot J, Torii M, Tsuboi T, Kumar N 2004. Potent immunogenicity of DNA vaccines encoding Plasmodium vivax transmission-blocking vaccine candidates Pvs 25 and Pvs28-evaluation of homologous and heterologous antigen-delivery prime-boost strategy. Vaccine 22: 3205-3213.

Kubler-Kielb J, Majadly F, Wu Y, Narum DL, Guo C, Miller LH, Shiloach J, Robbins JB, Schneerson R 2007. Long-lasting and transmission-blocking activity of antibodies to Plasmodium fal- ciparum elicited in mice by protein conjugates of Pfs25. Proc Natl Acad Sci USA 104: 293-298.

Lal AA, Patterson PS, Sacci JB, Vaughan JA, Paul C, Collins WE, Wirtz RA, Azad AF 2001. Anti-mosquito midgut antibodies block development of Plasmodium falciparum and Plasmodium vivax in multiple species of Anopheles mosquitoes and reduce vector fecundity and survivorship. Proc Natl Acad Sci USA 98: 5228-5233.

Lavazec C, Boudin C, Lacroix R, Bonnet S, Diop A, Thiberge S, Boisson B, Tahar R, Bourgouin C 2007. Carboxypeptidases B of Anopheles gambiae as targets for a Plasmodium falciparum transmission-blocking vaccine. Infect Immun 75: 1635-1642.

LeBlanc R, Vasquez Y, Hannaman D, Kumar N 2008. Markedly enhanced immunogenicity of a Pfs 25 DNA-based malaria transmission-blocking vaccine by in vivo electroporation. Vaccine 26: 185-192.

Lobo CA, Dhar R, Kumar N 1999. Immunization of mice with DNAbased Pfs 25 elicits potent malaria transmission-blocking antibodies. Infect Immun 67: 1688-1693.

Malkin EM, Durbin AP, Diemert DJ, Sattabongkot J, Wu Y, Miura K, Long CA, Lambert L, Miles AP, Wang J, Stowers A, Miller LH, Saul A 2005. Phase 1 vaccine trial of Pvs25H: a transmission blocking vaccine for Plasmodium vivax malaria. Vaccine 23: 3131-3138.

McMeniman CJ, Lane RV, Cass BN, Fong AW, Sidhu M, Wang YF, O'Neill SL 2009. Stable introduction of a life-shortening Wolbachia infection into the mosquito Aedes aegypti. Science 323: 141-144.

Milek RL, DeVries AA, Roeffen WF, Stunnenberg H, Rottier PJ, Konings RN 1998a. Plasmodium falciparum: heterologous synthesis of the transmission-blocking vaccine candidate Pfs $48 / 45$ in recombinant vaccinia virus-infected cells. Exp Parasitol 90: $165-174$.

Milek RL, Roeffen WF, Kocken CH, Jansen J, Kaan AM, Eling WM, Sauerwein RW, Konings RN 1998b. Immunological properties of recombinant proteins of the transmission blocking vaccine candidate, Pfs $48 / 45$, of the human malaria parasite Plasmodium falciparum produced in Escherichia coli. Parasite Immunol 20: 377-385.

Milek RL, Stunnenberg HG, Konings RN 2000. Assembly and expression of a synthetic gene encoding the antigen Pfs $48 / 45$ of the human malaria parasite Plasmodium falciparum in yeast. Vaccine 18: 1402-1411.

Oliveira F, Jochim RC, Valenzuela JG, Kamhawi S 2009. Sand flies, Leishmania and transcriptome-borne solutions. Parasitol Int 58: $1-5$.

Outchkourov N, Vermunt A, Jansen J, Kaan A, Roeffen W, Teelen K, Lasonder E, Braks A, van de Vegte-Bolmer M, Qiu LY, Sauerwein R, Stunnenberg HG 2007. Epitope analysis of the malaria surface antigen pfs $48 / 45$ identifies a subdomain that elicits transmission blocking antibodies. J Biol Chem 282: 17148-17156.

Outchkourov NS, Roeffen W, Kaan A, Jansen J, Luty A, Schuiffel D, van Gemert GJ, van de Vegte-Bolmer M, Sauerwein RW, Stunnenberg HG 2008. Correctly folded Pfs $48 / 45$ protein of Plasmodium falciparum elicits malaria transmission-blocking immunity in mice. Proc Natl Acad Sci USA 105: 4301-4305.

Quakyi IA, Carter R, Rener J, Kumar N, Good MF, Miller LH 1987. The 230-kDa gamete surface protein of Plasmodium falciparum is also a target for transmission-blocking antibodies. $J$ Immunol 139: 4213-4217.

Ramalho-Ortigao JM, Kamhawi S, Joshi MB, Reynoso D, Lawyer PG, Dwyer DM, Sacks DL, Valenzuela JG 2005. Characterization of a blood activated chitinolytic system in the midgut of the 
sand fly vectors Lutzomyia longipalpis and Phlebotomus papatasi. Insect Mol Biol 14: 703-712.

Saraiva EM, de Figueiredo Barbosa A, Santos FN, Borja-Cabrera GP, Nico D, Souza LO, de Oliveira Mendes-Aguiar C, de Souza EP, Fampa P, Parra LE, Menz I, Dias JG, Jr, de Oliveira SM, Palatnik-de-Sousa CB 2006. The FML-vaccine (Leishmune) against canine visceral leishmaniasis: a transmission blocking vaccine. Vaccine 24: 2423-2431.

Sattabongkot J, Tsuboi T, Hisaeda H, Tachibana M, Suwanabun N, Rungruang T, Cao YM, Stowers AW, Sirichaisinthop J, Coleman RE, Torii M 2003. Blocking of transmission to mosquitoes by antibody to Plasmodium vivax malaria vaccine candidates Pvs 25 and Pvs28 despite antigenic polymorphism in field isolates. $\mathrm{Am} J$ Trop Med Hyg 69: 536-541.

Saul A, Hensmann M, Sattabongkot J, Collins WE, Barnwell JW, Langermans JA, Wu Y, Long CA, Dubovsky F, Thomas AW 2007. Immunogenicity in rhesus of the Plasmodium vivax mosquito stage antigen Pvs25H with Alhydrogel and Montanide ISA 720. Parasite Immunol 29: 525-533

Singh M, O’Hagan D 1999. Advances in vaccine adjuvants. Nat Biotechnol 17: 1075-1081.

Thomas MB, Read AF 2007. Can fungal biopesticides control malaria? Nat Rev Microbiol 5: 377-383.

Titus RG, Bishop JV, Mejia JS 2006. The immunomodulatory factors of arthropod saliva and the potential for these factors to serve as vaccine targets to prevent pathogen transmission. Parasite Immunol 28: 131-141.
Tonui WK, Mbati PA, Anjili CO, Orago AS, Turco SJ, Githure JI, Koech DK 2001a. Transmission blocking vaccine studies in leishmaniasis: I. Lipophosphoglycan is a promising transmission blocking vaccine molecule against cutaneous leishmaniasis. East Afr Med J 78: 84-89.

Tonui WK, Mbati PA, Anjili CO, Orago AS, Turco SJ, Githure JI, Koech DK 2001b. Transmission blocking vaccine studies in leishmaniasis: II. Effect of immunisation using Leishmania major derived 63 kilodalton glycoprotein, lipophosphoglycan and whole parasite antigens on the course of $L$. major infection in BALB/c mice. East Afr Med J 78: 90-92.

Valenzuela JG 2004. Blood-feeding arthropod salivary glands and saliva. In WC Marquardt, Biology of disease vectors, Elsevier Academic Press, Burlington, p. 377-386.

van Dijk MR, Janse CJ, Thompson J, Waters AP, Braks JA, Dodemont HJ, Stunnenberg HG, van Gemert GJ, Sauerwein RW, Eling W 2001. A central role for P48/45 in malaria parasite male gamete fertility. Cell 104: 153-164.

Willadsen P 2004. Anti-tick vaccines. Parasitology 129 (Suppl.): S367-387.

Wu Y, Ellis RD, Shaffer D, Fontes E, Malkin EM, Mahanty S, Fay MP, Narum D, Rausch K, Miles AP, Aebig J, Orcutt A, Muratova O, Song G, Lambert L, Zhu D, Miura K, Long C, Saul A, Miller LH, Durbin AP 2008. Phase 1 trial of malaria transmission blocking vaccine candidates Pfs 25 and Pvs 25 formulated with montanide ISA 51. PLoS ONE 3: e2636. 